\title{
MicroRNA-22 Induces Endothelial Progenitor Cell Senescence by Targeting AKT3
}

\author{
Yue Zheng Zhiwei Xu \\ Department of Cardiothoracic Surgery, Shanghai Children's Medical Center, Shanghai Jiao Tong \\ University School of Medicine, Shanghai, China
}

\section{Key Words}

Endothelial progenitor cells $•$ Aging $•$ Senescence $•$ Angiogenesis $•$ AKT3

\begin{abstract}
Objectives: Endothelial progenitor cells (EPCs) play an important role in postnatal neovascularization. The number and function of EPCS declines as part of aging-associated senescence, thereby potentially contributing to vascular pathologies. Here, we investigated the significance and molecular mechanisms of microRNA-22 (miR-22) governing EPC senescence. Methods: EPCs were isolated from human circulating mononuclear cells from healthy young and aged volunteers. Cell senescence, proliferation, migration and tube formation ability were detected by SA- $\beta$-gal staining assay, MTT assay, transwell assay and Matrigel-based angiogenesis assay. Gene and protein expression were analyzed by qRT-PCR and Western blot respectively. Results: We found that miR-22 was upregulated in aged EPCs. Overexpression of miR-22 in young EPCs induced cell senescence, decreased proliferation and migration, and impaired angiogenesis in vitro. Conversely, silencing of endogenous miR-22 led to decreased cell senescence, increased proliferation and migration, and improved angiogenesis. AKT3 was identified as a direct target of miR-22, and restoration of AKT3 expression attenuated the effects of miR-22 in young EPCs. Conclusion: Our results indicate that miR-22 induces EPC senescence by downregulating AKT3 expression, providing a potential novel target for the reversal of EPC dysfunction in angiogenesis.
\end{abstract}

Copyright $\odot 2014$ S. Karger AG, Basel

\section{Introduction}

An imbalance of angiogenesis contributes to numerous disorders such as ischemia and inflammation [1]. Endothelial progenitor cells (EPCs) play a prominent role in vascular morphogenesis, both during embryonic development and in postnatal neovascularization, 
where they contribute to tissue maintenance and repair in various pathological conditions $[2,3]$. In the adult, EPCs are mobilized from the bone marrow (BM) and peripheral blood (PB) into the circulation in response to growth factors and cytokines released following stimuli such as vascular trauma [4], ischemia [5], and wounding [6]. However, aged EPCs are less able to contribute to vascular repair and regeneration and thereby contribute to the increased propensity toward vascular pathology in normal human aging [7]. Reduced EPC number and deregulated EPC function are observed in pathological conditions, including atherosclerosis [3], vascular intima hyperplasia [8], ischemic heart disease [9], and graft vasculopathy [10]. Understanding EPC biology is therefore essential for developing therapeutic applications to improve neovascularization in non-neoplastic disease.

Cellular aging or senescence is characterized by cell cycle arrest and an accumulation of CDK inhibitors such as $\mathrm{p} 16^{\mathrm{INK} 4 \mathrm{a}}$ [11]. Various signaling pathways trigger senescence in response to oxidative stress, telomere erosion, replicative stress or genotoxic stress [11, 12]. The molecular mechanisms underlying the heterogeneous triggers and phenotypes of senescence are complex and the recent identification of senescence associated microRNAs (miRNAs) has added another facet to the control of senescence.

miRNAs are a class of naturally occurring small non coding RNAs that negatively regulate the stability and translation of target protein-coding mRNAs at the $3^{\prime}$ untranslated region (UTR). miRNAs often target a cluster of genes, allowing them to regulate a variety of biological processes including cell proliferation, differentiation and apoptosis $[13,14]$. A number of miRNAs have been identified as regulators of new vessel formation by endothelial cells during angiogenesis $[15,16]$, including the proangiogenic miRNAs let-7f, miR-27b, the miR-17-92 cluster and miR-126, and the antiangiogenic miRNAs miR-221/miR-222, miR-34 and miR-217 $[17,18]$. However, the role of miRNAs in EPC function has not been extensively explored. A recent miRNA profiling and microanalysis identified miR-10A* and miR-21 as modulators of EPC senescence in mice [19]. The same study reported an upregulation of miR-22 in aged EPCs [19]. In the present study, we show that miR-22 is upregulated in aged human EPCs and investigate the possible role of miR-22 in EPC function in vitro. We identified the signaling molecule AKT3 as a direct and functional target of miR-22 mediating its role in EPC angiogenic function, providing a potential therapeutic target for the reversal of EPC dysfunction in vascular pathologies.

\section{Materials and Methods}

\section{Isolation and culture of EPCS}

Human EPCs were isolated from peripheral blood obtained from six healthy donors. The procedure was approved by the Clinical Research Ethics Committee of Shanghai Jiaotong University, and informed consent was obtained from all donors. The blood samples were separated into two groups (n=3): a young group with an average age of $21 \pm 6$ years and an aged group with an average age of $66 \pm 7$ years. EPCs were isolated and cultured as previously described [20]. In brief, total mononuclear cells (MNCs) from $40 \mathrm{ml}$ of peripheral blood were isolated using Ficoll density-gradient centrifugation, plated on fibronectin-coated six-well plates and cultured in endothelial basal medium (EBM, Cambrex, Charles, IA) supplemented with human vascular endothelial growth factor (VEGF), R3-insulin-like growth factor 1, ascorbic acid, cortisol, human fibroblast growth factor, gentamicin, amphotericin and $20 \%$ fetal bovine serum at $37^{\circ} \mathrm{C}$ in a $5 \% \mathrm{CO}_{2}$ incubator. After 7 days of culture, attached early EPCs developed a spindle-shaped appearance. These cells can grow into colonies after 2-4 weeks, and exhibit a "cobblestone" morphology and monolayer growth pattern typical of mature endothelial cells at confluence. EPCs at early passages (passage 2 to 3 ) were used for the phenotypic and functional analyses.

\section{Lentivirus production and transduction}

The lentivectors encoding miR-22, anti-miR-22 and their respective controls, and AKT3 open reading frame (ORF) with or without $3^{\prime}$-UTR were purchased from Hanbio (Shanghai, China). Lentivirus was produced by co-transfecting these lentivectors individually with the packaging plasmids into 293T cells 
using Lipofectamine 2000 (Invitrogen, Carlsbad, CA). EPCs were seeded into six-well dishes $\left(5 \times 10^{5}\right.$ per well), and infected with the recombinant lentivirus-transducing units. After $48 \mathrm{~h}$, cells were selected by culture in the presence of puromycin for one week.

RNA isolation and quantitative real time polymerase chain reaction ( $q R T-P C R$ )

Total RNA was extracted with the TRIzol reagent (Invitrogen) and treated with DNase I (Fermentas, Ottawa, Canada) to remove any contaminating genomic DNA. The concentration and quality of RNA were evaluated using spectrophotometry and denaturing gel electrophoresis. Complementary DNA was synthesized using a PrimeScript RT reagent kit (Bio-Rad Laboratories, Hercules, CA) according to the manufacturer's instructions. Mature miR-22 expression was measured and quantified using the miRNAspecific TaqMan miRNA assay kit (Applied Biosystems, Foster City, CA). For mRNA, qRT-PCR was performed using a Fast Start Master SYBR Green Kit (Roche, Basel, Switzerland). The analyses were carried out on the ABI 7900HT Sequence Detection System (Applied Biosystems). Expression levels of miRNA and mRNA were calculated using the $2^{-\Delta \Delta \mathrm{CT}}$ method, with $\mathrm{U} 6$ and $\beta$-actin as endogenous controls. All reactions were run in triplicate.

\section{Senescence-associated beta-galactosidase (SA- $\beta$-gal) staining}

EPC senescence was determined using a SA- $\beta$-gal staining kit (Beyotime, China). Cells were fixed for 15 min at room temperature in fixation buffer, washed with PBS and incubated with the working solution for $12 \mathrm{~h}$ at $37^{\circ} \mathrm{C}$ without $\mathrm{CO}_{2}$. PBS was added to stop the reaction. The ration of $\beta$-gal-positive cells to the total number of cells was determined.

\section{Cell proliferation and cell cycle analyses}

EPC viability was determined using the MTT assay. Lentiviral infected cells were seeded in 96-well plates for $96 \mathrm{~h}$. Cells were stained with $100 \mu \mathrm{l}$ of MTT stock solution $(0.5 \mathrm{mg} / \mathrm{ml})$ for $4 \mathrm{~h}$ at $37^{\circ} \mathrm{C}$, followed by removal of the culture medium and addition of $150 \mu \mathrm{l}$ dimethyl sulfoxide (DMSO; Sigma, St. Louis, MO). The absorbance at a wavelength of $540 \mathrm{~nm}$ was measured to calculate the number of viable cells. For cell cycle analysis, cells were fixed in $70 \%$ ethanol at $4^{\circ} \mathrm{C}$ for $24 \mathrm{~h}$, stained with $50 \mu \mathrm{g} / \mathrm{ml}$ propidium iodide solution, and analyzed using a FACSCalibur flow cytometer (BD Bioscience, Bedford, MD). The experiment was repeated at least three times.

\section{Migration assay}

EPC migration was evaluated using a modified Boyden chamber assay. Cells $\left(4 \times 10^{4}\right)$ were suspended in EBM and loaded into the upper chamber of the insert ( $8 \mu \mathrm{m}$ pore size, BD Biosciences). The lower chamber was filled with EBM supplemented with VEGF $(50 \mathrm{ng} / \mathrm{ml})$. After $24 \mathrm{~h}$ of incubation, the cells migrated through the pores were fixed, stained with crystal violet, and counted with an IX71 inverted microscope (Olympus, Tokyo, Japan). Analyses were performed in triplicate.

\section{Tube formation assay}

An in vitro tube formation assay was performed to assess the angiogenic capacity of EPCs [20]. In brief, Matrigel (BD Biosciences) was thawed at $4^{\circ} \mathrm{C}$ overnight, aliquoted into a pre-cooled 96-well plate and incubated at $37^{\circ} \mathrm{C}$ for $45 \mathrm{~min}$. EPCs $\left(1 \times 10^{4}\right)$ were plated onto the preincubated Matrigel and incubated at $37^{\circ} \mathrm{C}$ for $12-15 \mathrm{~h}$. Images of tube morphology were recorded in 10 random microscopic fields per sample. Tube formation was defined as a tube-like structure exhibiting a length four times its width. The cumulative tube lengths were examined by Image-Pro Plus software (Media Cybernetics, Silver Spring, MA).

\section{Luciferase assays}

An AKT3 3'-UTR fragment containing potential miR-22 binding site was cloned into the pGL3-basic vector (Promega, Madison, WI). The corresponding mutant construct, pGL3-AKT-mut, was created by mutating the miR-22 binding site using a QuickChange Site-Directed Mutagenesis Kit (Agilent Technologies, Palo Alto, CA). 293T cells infected with miR-22 lentivirus or anti-miR-22 lentivirus were seeded into 96-well plates. An aliquot containing 100 ng pGL3-AKT-wt or pGL3-AKT-mut was cotransfected with 20 ng pRLTK vector into these cells by Lipofectamine 2000. After transfection for $48 \mathrm{~h}$, Firefly luciferase and Renilla luciferase luminescence was measured using the Dual-Glo luciferase kit (Promega). 
Western blot analysis

Total proteins were extracted using RIPA buffer and protein concentrations were analyzed using the BCA protein assay kit (Beyotime). Protein lysates were resolved by SDS-PAGE and transferred to polyvinylidene difluoride (PVDF) membranes. The membranes were probed with specific antibodies against p16, AKT3 and $\beta$-actin (Cell Signaling Technology, Inc., MA), followed by the appropriate peroxidaseconjugated secondary antibody. Signals were detected using the enhanced chemiluminescence reagents (Pierce Biotechnology, Rockford, IL).

\section{Statistical analysis}

Data were expressed as the mean \pm SD from at least 3 independent experiments. Student $t$ test was used to assess differences. $P$ values $<0.05$ were considered to be significant.

\section{Results}

miR-22 is upregulated in aged EPCs

miR-22 is differentially expressed in lineage negative (lin-) bone marrow cells (BMCs) from young and old mice [19], indicating its potential role in EPC senescence. To determine whether human EPCs displayed the same temporal miR-22 expression profile as in mice, we investigated miR-22 expression in young and aged human EPCs. Analysis by qRT-PCR showed that miR-22 is expressed at low levels in young EPCs (Y-EPCs) and upregulated in aged EPCs (A-EPCs) (Fig. 1A).

miR-22 regulates EPC senescence and proliferation

To determine whether miR-22 plays a role in EPC senescence, we overexpressed miR-22 in Y-EPCs or knocked down miR-22 expression with anti-miR-22 in A-EPCs (Fig. 1B). Using $\beta$-galactosidase expression as an indicator of cell senescence, we found that overexpression of miR-22 in Y-EPCs increased senescence whereas its depletion decreased A-EPC senescence (Fig. 1C). Consistent with this result, overexpression of miR-22 in Y-EPCs upregulated p16 $16^{\mathrm{INK} 4 \mathrm{~A}}$, a senescence marker. By contrast, knockdown of miR-22 in A-EPCs downregulated p16 ${ }^{\mathrm{INK} 4 \mathrm{~A}}$ expression (Fig. 1D). Moreover, we investigated whether miR-22 affects EPC proliferation by MTT assay. The results showed that overexpression of miR-22 in Y-EPCs inhibited proliferation whereas knockdown of miR-22 in A-EPCs increased their proliferation (Fig. 1E). Collectively, these data indicate that miR-22 inhibits proliferation and promotes senescence in EPCs.

miR-22 impairs in vitro migration and angiogenesis

Migration is an integral function of EPCs in angiogenesis. As shown in Fig. 2A, overexpression of miR-22 reduced Y-EPC migration whereas anti-miR-22 increased A-EPC migration. Also the role of miR-22 in angiogenic tubule formation by seeding on Matrigel substratum Y-EPCs overexpressing miR-22 or A-EPCs with reduced mir-22 were investigated. miR-22 overexpression inhibited tube formation in Y-EPCs, whereas miR-22 knock-down had the opposite effect on A-EPCs (Fig. 2B). These results suggest an anti-angiogenic role for miR-22.

AKT3 is a direct target of miR-22

While searching for potential targets of miR-22 using available databases such as TargetScan, miRanda and PicTar, AKT3 has been chosen for its central role in the PI3K/ AKT signaling pathway, which besides its roles in multiple cellular functions, also impacts senescence [21]. Analysis of the 3'UTR sequence of AKT3 identified a possible binding site for miR-22 (Fig. 3A). To test the function of this potential binding site, we co-expressed a wild-type or mutant AKT3 3'UTR sequence downstream of the luciferase reporter gene with either miR-22 or miR-Ctrl in Y-EPCs. As shown in Fig. 3B, miR-22 overexpression decreased the relative luciferase activity of the $3^{\prime} \mathrm{UTR}$ reporter, whereas it had no effect on the mutant 


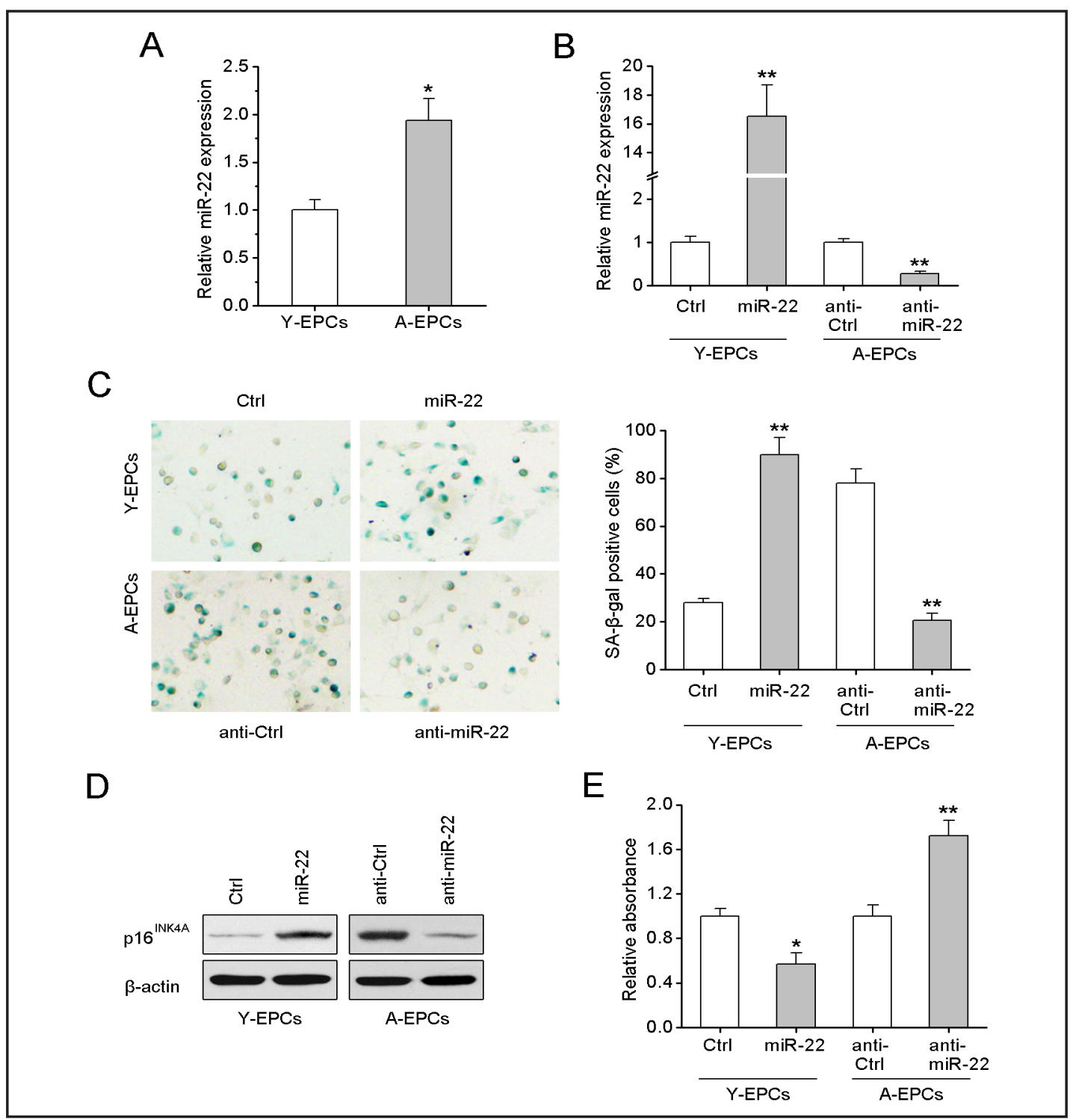

Fig. 1. miR-22 modulates senescence and proliferation in young (Y)- and aged (A)-EPCs in vitro. (A) The expression of miR-22 detected by qRT-PCR in Y- and A-EPCs. qRT-PCR analysis of miR-22 expression (B), representative photos and quantitative analysis of senescence-associated $\beta$-galactosidase (SA- $\beta$-gal) staining (C), Western blot analysis of p16 expression (D) and cell viability as determined by MTT assay (F) in Y- and A-EPCs infected with lentivirus encoding miR-22, anti-miR-22 or the corresponding control. ${ }^{*} P<0.05$, ${ }^{* *} P<0.01$.

$3^{\prime}$ UTR reporter, indicating that the intact seed sequence is required for miR-22 functionality. Furthermore, miR-22 overexpression in Y-EPCs significantly downregulated AKT3 mRNA and protein expression, whereas miR-22 knockdown in A-EPCs increased AKT3 mRNA and protein levels (Fig. 3C and D). Taken together, these results indicate that miR22 directly targets and downregulates AKT3 in EPCs.

\section{Re-introduction of AKT3 rescues the effects of miR-22}

To ascertain whether the effects of miR-22 gain- and loss-of-function in EPCs are mediated by the downregulation of AKT3 by miR-22, we co-expressed miR-22 and AKT3 with or without its 3'UTR in Y-EPCs (Fig. 4A). Co-expression of the AKT3-3'UTR with miR22 maintains high p16 protein levels. However, removal of the $3^{\prime} \mathrm{UTR}$ resulted in decreased 

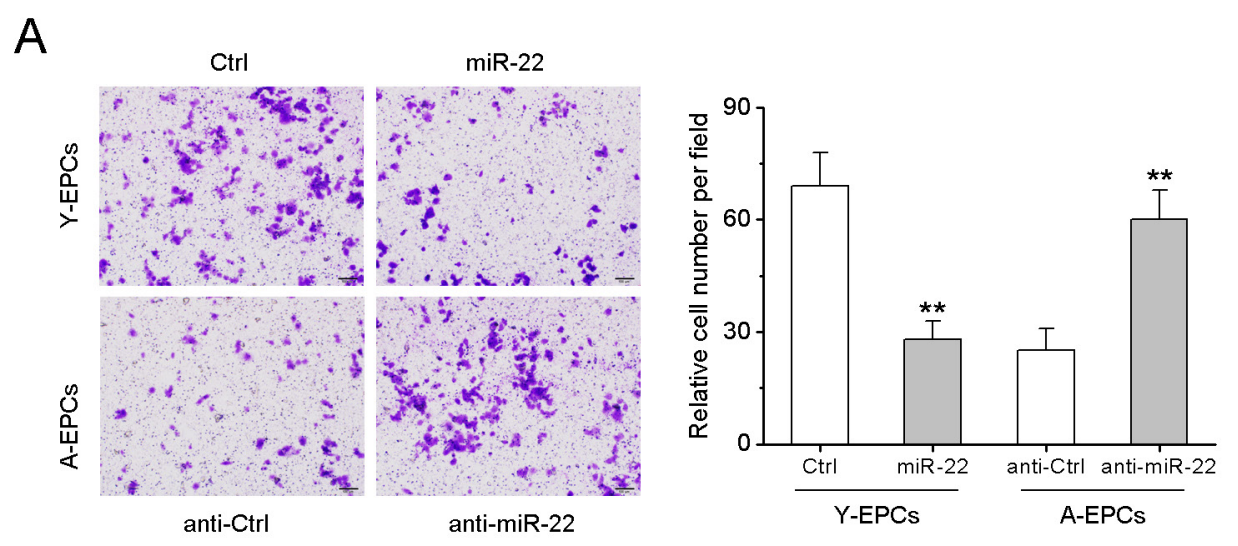

B
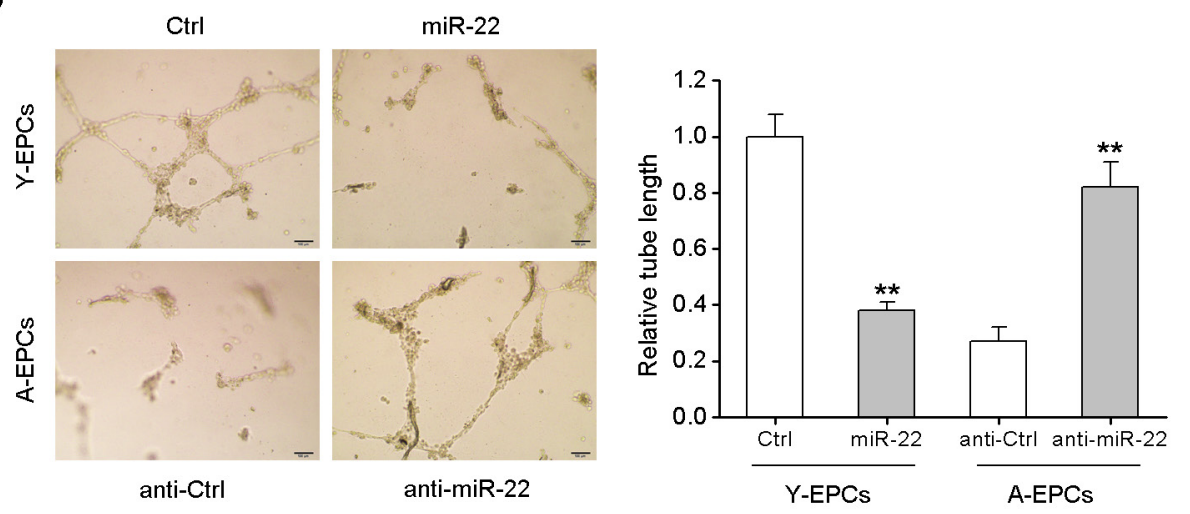

Fig. 2. miR-22 affects EPC migration and angiogenesis in vitro. (A) Representative images (left) of the Transwell assay and quantitation (right) of cell migration of Y-EPCs infected with lentivirus encoding miR22 or Ctrl and A-EPCs infected with lentivirus encoding anti-miR-22 or anti-Ctrl. (B) Representative images of the vascular tube formation assay (left) and quantitation of cumulative tube lengths in the endothelial network (right) of Y-EPCs infected with lentivirus encoding miR-22 or miR-Ctrl and A-EPCs infected with lentivirus encoding anti-miR-22 or anti-Ctrl. ${ }^{* *} P<0.01$.

p16 protein levels (Fig. 4A). Using the same experimental design, we showed that the miR22 promotion of senescence and repression of cell viability, migration and vascular tube formation depends on the presence of an AKT3 transcript with an intact 3'UTR (Fig. 4B-E).

\section{Discussion}

The major new findings of this study were that miR-22 expression in EPCs increases with aging, and its overexpression promotes cell senescence but inhibits proliferation, migration and angiogenesis by inhibiting the serine/threonine kinase AKT3, one of three isoforms of AKT (also termed protein kinase B, PKB) [22].

The role of miR-22 in cell proliferation and senescence appears to be highly context dependent. It has been shown that miR-22 induces senescence of neonatal rat cardiac fibroblasts by modulating the expression of mimecan (osteoglycin, OGN) [23]. In cancer cell lines, miR-22 inhibits cell proliferation by targeting MYCBP (c-Myc-binding protein) [24], p21(CDKN1A) [25], ErbB3 [26], TIAM1 (T-cell lymphoma invasion and metastasis 1) [27] and $\mathrm{Sp} 1$ [28] and restores cellular senescence by targeting the senescence-associated genes CDK6, SIRT1 and Sp1 [29]. Conversely, miR-22 promotes tumorigenesis and metastasis by directly targeting the PI3K/AKT inhibitor PTEN (phosphatase tensin and homolog) [30, 
A

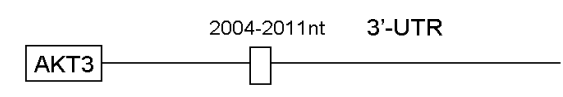

5'. GgGCAUUUUACUAAGGCAGCUA . 3' AKT3-3'-UTR

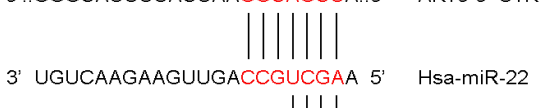

\|\|

5'..GGGCAUUUUACUAACCGAGCUA..3' AKT3-3'UTR-MUT

C

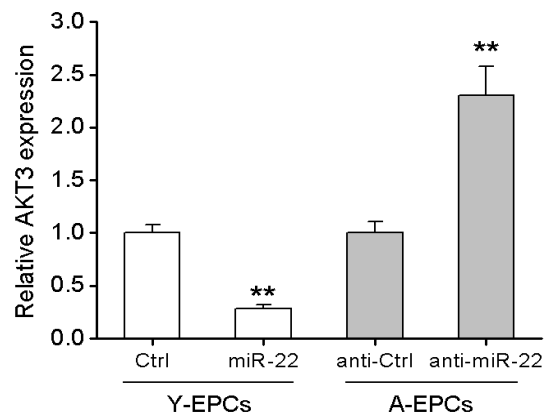

B

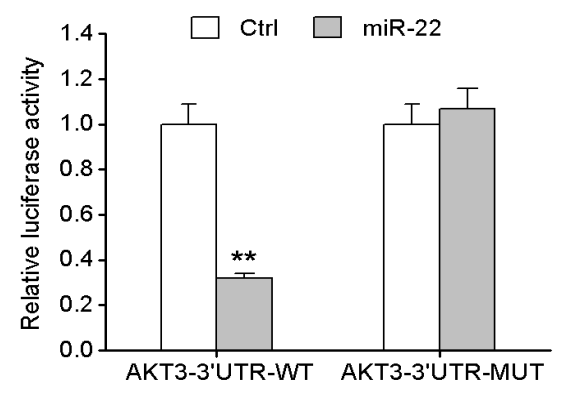

D

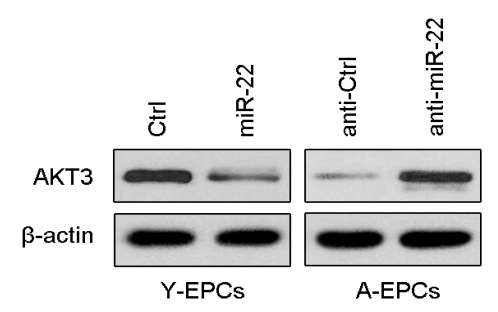

Fig. 3. miR-22 downregulates AKT3 expression by directly targeting its $3^{\prime}$-UTR. (A) Diagram showing the seed sequences of miR-22 and its predicted binding site in the $3^{\prime}$-UTR of AKT3. (B) Luciferase reporter assays in Y-EPCs co-transfected with wt or mut 3'-UTR and miRNAs as indicated. (C) AKT3 mRNA expression levels determined by qRT-PCR. (D) AKT3 protein expression levels detected by Western blotting. ${ }^{* *} P<0.01$.
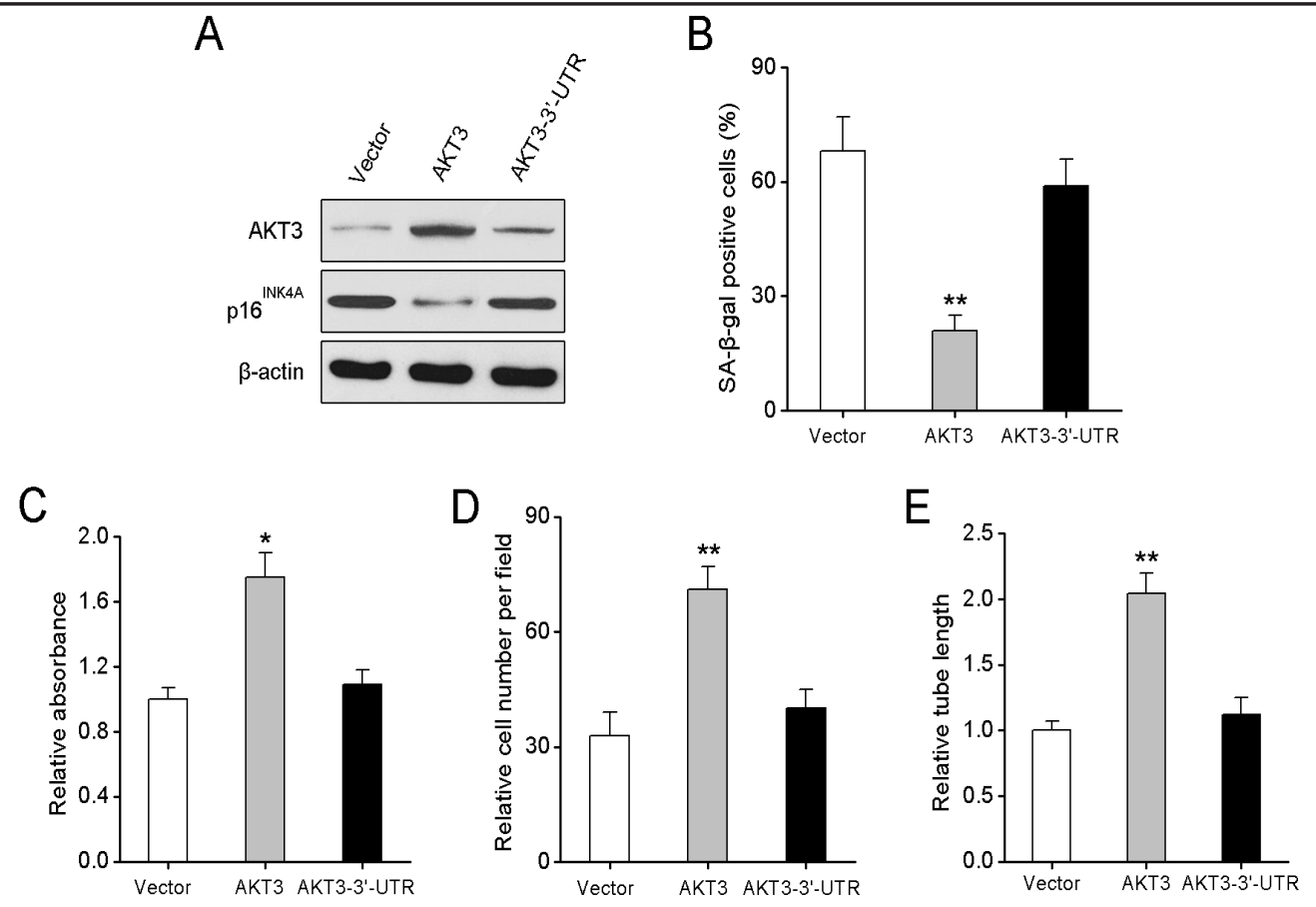

Fig. 4. Re-introduction of AKT3 without its $3^{\prime}$-UTR reverses the effects of miR-22 overexpression. (A) Western blot analysis of the levels of AKT3 and p16 in Y-EPCs co-infected with lentivirus encoding miR-22 and AKT3 with or without its 3'-UTR. Overexpression of AKT3 without its 3 '-UTR attenuated the effects of miR-22 on SA- $\beta$-gal staining (B), cell viability (C), migration (D) and vascular tube formation (E). ${ }^{*} P<0.05$, $* * P<0.01$. 
31] and the TET (Ten eleven translocation) family of methylcytosine dioxygenases [32, 33]. In the present study, we showed for the first time that miR-22 expression is upregulated in association with aging. Overexpression of miR-22 in young EPCs promoted senescence and inhibited proliferation, whereas its depletion suppressed senescence and promoted proliferation in aged EPCs. Furthermore, we showed that miR-22 overexpression suppressed EPC migration and angiogenesis. These data indicate that miR-22 overexpression induces senescence in EPCs and may thereby result in their impaired angiogenic function.

We identified AKT3 as a direct target of miR-22 and showed that miR-22 overexpression significantly upregulated AKT3 mRNA and protein levels by directly targeting its $3^{\prime}$ UTR in young EPCs. AKT is a central protein mediating signals from receptor tyrosine kinases and phosphatidylinositol 3-kinase (PI3K). As a key component of the PI3K/AKT pathway, AKT regulates signaling of multiple biological and pathophysiological processes $[22,34]$. Thus, it is conceivable that miR-22's inhibition of AKT3 impinges on a range of PI3K/AKT pathway-driven functions in EPCs, including proliferation, angiogenesis, migration or even the regulation of longevity in the insulin/IGF-1 signaling pathway [35].

One of the hallmarks of miRNA is its ability to target genes involved in multiple biological pathways. In keeping with this feature, miR-22 is able to inhibit as well as activate the PI3K/AKT pathway, itself a complex hub for multiple essential signaling pathways. The miR-22 activation of the PI3K/AKT pathway by PTEN inhibition leads to tumorigenesis and metastasis [30,31], whereas its inhibition of the pathway by AKT3 inhibition leads to reduced proliferation and migration. The results of the present study confirm the role of miR-22 as an inhibitor of multiple targets in diverse biological pathways and provide a novel therapeutic target for the reversal of EPC dysfunction in angiogenesis.

\section{References}

1 Carmeliet P: Angiogenesis in life, disease and medicine. Nature 2005;438:932-936.

-2 Khoo CP, Pozzilli P, Alison MR: Endothelial progenitor cells and their potential therapeutic applications. Regenerative medicine 2008;3:863-876.

3 Fadini GP, Losordo D, Dimmeler S: Critical reevaluation of endothelial progenitor cell phenotypes for therapeutic and diagnostic use. Circ Res 2012;110:624-637.

4 Gill M, Dias S, Hattori K, Rivera ML, Hicklin D, Witte L, Girardi L, Yurt R, Himel H, Rafii S: Vascular trauma induces rapid but transient mobilization of vegfr2(+)ac133(+) endothelial precursor cells. Circ Res 2001;88:167-174.

5 Takahashi T, Kalka C, Masuda H, Chen D, Silver M, Kearney M, Magner M, Isner JM, Asahara T: Ischemia- and cytokine-induced mobilization of bone marrow-derived endothelial progenitor cells for neovascularization. Nature Med 1999;5:434-438.

6 Lyden D, Hattori K, Dias S, Costa C, Blaikie P, Butros L, Chadburn A, Heissig B, Marks W, Witte L, Wu Y, Hicklin D, Zhu Z, Hackett NR, Crystal RG, Moore MA, Hajjar KA, Manova K, Benezra R, Rafii S: Impaired recruitment of bone-marrow-derived endothelial and hematopoietic precursor cells blocks tumor angiogenesis and growth. Nature Med 2001;7:1194-1201.

7 Toda N: Age-related changes in endothelial function and blood flow regulation. Pharmacol Ther 2012;133:159-176.

8 Zhang J, Zhang Z, Zhang DY, Zhu J, Zhang T, Wang C: microRNA 126 inhibits the transition of endothelial progenitor cells to mesenchymal cells via the PIK3R2-PI3K/Akt signalling pathway. PLoS One 2013;8:e83294.

-9 Schmidt-Lucke C, Rossig L, Fichtlscherer S, Vasa M, Britten M, Kamper U, Dimmeler S, Zeiher AM: Reduced number of circulating endothelial progenitor cells predicts future cardiovascular events: Proof of concept for the clinical importance of endogenous vascular repair. Circulation 2005;111:2981-2987.

10 Hoeben A, Landuyt B, Highley MS, Wildiers H, Van Oosterom AT, De Bruijn EA: Vascular endothelial growth factor and angiogenesis. Pharmacol Rev 2004;56:549-580.

11 Chandler H, Peters G: Stressing the cell cycle in senescence and aging. Curr Opin Cell Biol 2013;25:765-771.

$>12$ Salama R, Sadaie M, Hoare M, Narita M: Cellular senescence and its effector programs. Genes Dev 2014;28:99-114.

13 Bartel DP: Micrornas: Genomics, biogenesis, mechanism, and function. Cell 2004;116:281-297. 


\section{Cellular Physiology Cell Physiol Biochem 2014;34:1547-1555

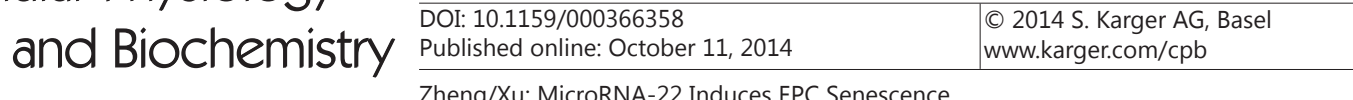

14 He L, Hannon GJ: Micrornas: Small rnas with a big role in gene regulation. Nat Rev Genet 2004;5:522-531.

15 Suarez Y, Sessa WC: Micrornas as novel regulators of angiogenesis. Circul Res 2009;104:442-454.

-16 Urbich C, Kuehbacher A, Dimmeler S: Role of micrornas in vascular diseases, inflammation, and angiogenesis. Cardiovasc Res2008;79:581-588.

17 Menghini R, Casagrande V, Cardellini M, Martelli E, Terrinoni A, Amati F, Vasa-Nicotera M, Ippoliti A, Novelli G, Melino G, Lauro R, Federici M: Microrna 217 modulates endothelial cell senescence via silent information regulator 1. Circulation 2009;120:1524-1532.

18 Zhao T, Li J, Chen AF: Microrna-34a induces endothelial progenitor cell senescence and impedes its angiogenesis via suppressing silent information regulator 1. Am J Physiol Endocrinol Metabol 2010;299:E110-116.

19 Zhu S, Deng S, Ma Q, Zhang T, Jia C, Zhuo D, Yang F, Wei J, Wang L, Dykxhoorn DM, Hare JM, GoldschmidtClermont PJ, Dong C: Microrna-10a* and microrna-21 modulate endothelial progenitor cell senescence via suppressing high-mobility group a2. Circul Res 2013;112:152-164.

20 Meng S, Cao JT, Zhang B, Zhou Q, Shen CX, Wang CQ: Downregulation of microrna-126 in endothelial progenitor cells from diabetes patients, impairs their functional properties, via target gene spred-1. J Mol Cell Cardiol 2012;53:64-72.

-21 Kang SS, Kwon T, Kwon DY, Do SI: Akt protein kinase enhances human telomerase activity through phosphorylation of telomerase reverse transcriptase subunit. J Biol Chem 1999;274:13085-13090.

22 Scheid MP, Woodgett JR: Unravelling the activation mechanisms of protein kinase b/akt. FEBS Lett 2003;546:108-112.

-23 Jazbutyte V, Fiedler J, Kneitz S, Galuppo P, Just A, Holzmann A, Bauersachs J, Thum T: Microrna-22 increases senescence and activates cardiac fibroblasts in the aging heart. Age 2013;35:747-762.

24 Xiong J, Du Q, Liang Z: Tumor-suppressive microrna-22 inhibits the transcription of e-box-containing c-myc target genes by silencing c-myc binding protein. Oncogene 2010;29:4980-4988.

25 Tsuchiya N, Izumiya M, Ogata-Kawata H, Okamoto K, Fujiwara Y, Nakai M, Okabe A, Schetter AJ, Bowman ED, Midorikawa Y, Sugiyama Y, Aburatani H, Harris CC, Nakagama H: Tumor suppressor mir-22 determines p53-dependent cellular fate through post-transcriptional regulation of p21. Cancer Res 2011;71:46284639.

26 Ling B, Wang GX, Long G, Qiu JH, Hu ZL: Tumor suppressor mir-22 suppresses lung cancer cell progression through post-transcriptional regulation of erbb3. J Cancer Res Clin Oncol 2012;138:1355-1361.

-27 Li B, Song Y, Liu TJ, Cui YB, Jiang Y, Xie ZS, Xie SL: Mirna-22 suppresses colon cancer cell migration and invasion by inhibiting the expression of t-cell lymphoma invasion and metastasis 1 and matrix metalloproteinases 2 and 9. Oncol Rep2013;29:1932-1938.

28 Guo MM, Hu LH, Wang YQ Chen P, Huang JG, Lu N, He JH, Liao CG: Mir-22 is down-regulated in gastric cancer, and its overexpression inhibits cell migration and invasion via targeting transcription factor sp1. Medical Oncol 2013;30:542.

29 Xu D, Takeshita F, Hino Y, Fukunaga S, Kudo Y, Tamaki A, Matsunaga J, Takahashi RU, Takata T, Shimamoto A, Ochiya T, Tahara H: Mir-22 represses cancer progression by inducing cellular senescence. J Cell Biol 2011;193:409-424.

-30 Gurha P, Abreu-Goodger C, Wang T, Ramirez MO, Drumond AL, van Dongen S, Chen Y, Bartonicek N, Enright AJ, Lee B, Kelm RJ, Jr., Reddy AK, Taffet GE, Bradley A, Wehrens XH, Entman ML, Rodriguez A: Targeted deletion of microrna-22 promotes stress-induced cardiac dilation and contractile dysfunction. Circulation 2012;125:2751-2761.

-31 Poliseno L, Salmena L, Riccardi L, Fornari A, Song MS, Hobbs RM, Sportoletti P, Varmeh S, Egia A, Fedele G, Rameh L, Loda M, Pandolfi PP: Identification of the mir-106b 25 microrna cluster as a proto-oncogenic pten-targeting intron that cooperates with its host gene mcm7 in transformation. Science Signal 2010;3:ra29.

-32 Song SJ, Ito K, Ala U, Kats L, Webster K, Sun SM, Jongen-Lavrencic M, Manova-Todorova K, Teruya-Feldstein J, Avigan DE, Delwel R, Pandolfi PP: The oncogenic microrna mir-22 targets the tet2 tumor suppressor to promote hematopoietic stem cell self-renewal and transformation. Cell Stem Cell 2013;13:87-101.

-33 Song SJ, Poliseno L, Song MS, Ala U, Webster K, Ng C, Beringer G, Brikbak NJ, Yuan X, Cantley LC, Richardson AL, Pandolfi PP: Microrna-antagonism regulates breast cancer stemness and metastasis via tet-familydependent chromatin remodeling. Cell 2013;154:311-324.

-34 Bellacosa A, Kumar CC, Di Cristofano A, Testa JR: Activation of akt kinases in cancer: Implications for therapeutic targeting. Adv Cancer Res 2005;94:29-86.

-35 Kloet DE, Burgering BM: The pkb/foxo switch in aging and cancer. Biochim Biophys Acta 2011;1813:19261937. 